\title{
Transitive eggs
}

\author{
John Bamberg Tim Penttila
}

full screen

close

quit

\begin{abstract}
We prove that a pseudo-oval or pseudo-ovoid (that is not an oval or ovoid) admitting an insoluble transitive group of collineations is elementary and arises over an extension field from a conic, an elliptic quadric, or a Suzuki-Tits ovoid.
\end{abstract}

Keywords : pseudo-oval, pseudo-ovoid, egg, translation generalised quadrangle, transitive

MSC 2000: 51E20

\section{Introduction}

An egg of the projective space $\mathrm{PG}(2 n+m-1, q)$ is a set $\mathcal{E}$ of $q^{m}+1$ subspaces of dimension $(n-1)$ such that every three are independent (i.e., span a $(3 n-1)$-dimensional subspace), and such that each element of $\mathcal{E}$ is contained in a common complement to the other elements of $\mathcal{E}$ (i.e., each element of $\mathcal{E}$ is contained in an $(n+m-1)$-dimensional subspace having no point in common with any other element of $\mathcal{E}$ ). The theory of eggs is equivalent to the theory of translation generalised quadrangles (see [20, Chapter 8]). If $q$ is even, then $m=n$ or $m=2 n$ (see [20, 8.7.2]), and for $q$ odd, the only known examples of eggs have $m=n$ or $m=2 n$. Now an ovoid of PG $(3, q)$ is an example of an egg where $m=2 n=1$; hence an egg having $m=2 n$ is called a pseudo-ovoid. Likewise, an oval of $\operatorname{PG}(2, q)$ is an egg where $m=n=2$, and henceforth, a pseudo-oval is an egg with $m=n$. If $\mathcal{O}$ is an oval of $\operatorname{PG}\left(2, q^{n}\right)$, then by field reduction from $\operatorname{GF}\left(q^{n}\right)$ to $\operatorname{GF}(q)$, one obtains a pseudo-oval of $\operatorname{PG}(3 n-1, q)$. Such pseudo-ovals are called elementary. Likewise, field reduction of an ovoid of $\mathrm{PG}\left(3, q^{n}\right)$ yields an elementary pseudo-ovoid of $\mathrm{PG}(4 n-1, q)$. All known pseudo-ovals are elementary, and in even characteristic, every known example of a pseudo-ovoid is elementary. There is some conflict over the definition of a 



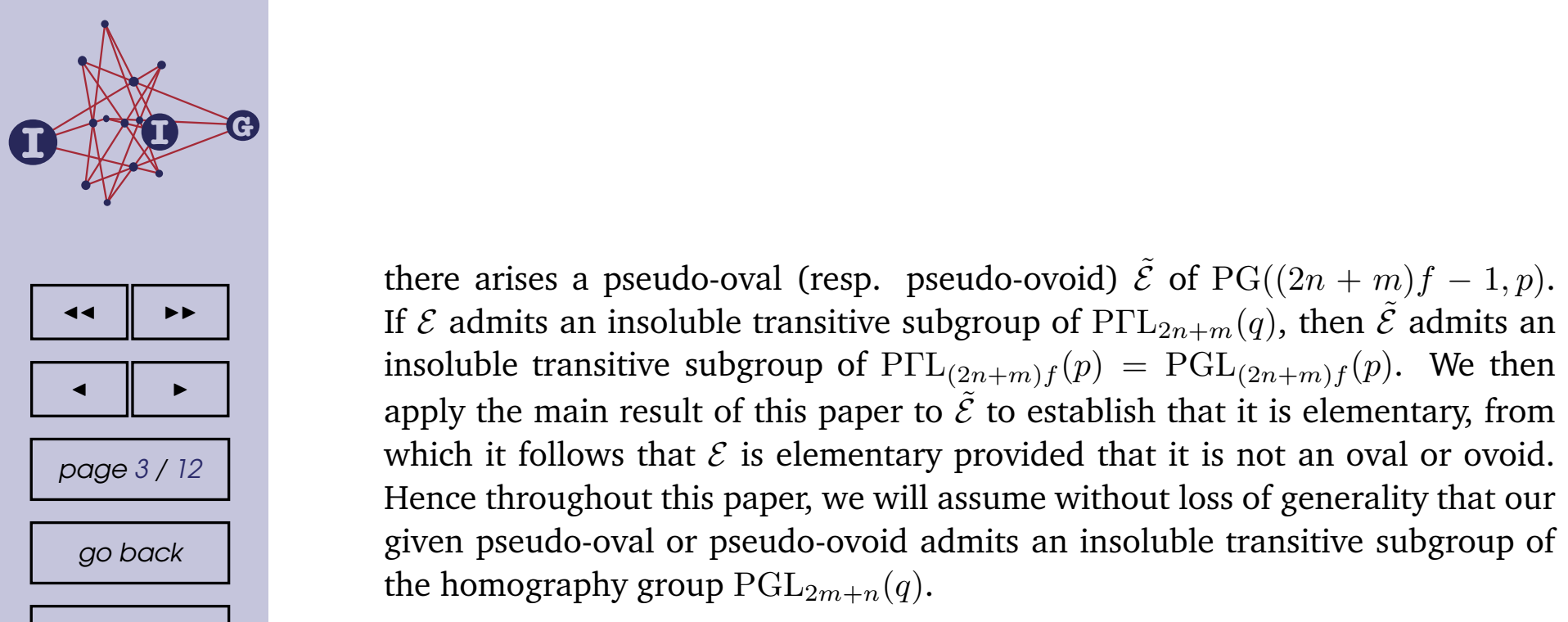

full screen

close

\section{The pseudo-oval case}

quit

A pseudo-oval of $\operatorname{PG}(d-1, q)$ (where $d$ is a multiple of 3 ) is a set of $q^{e / 2}+1$ subspaces of dimension $d / 3-1$, where $e=\frac{2}{3} d$. This phrasing makes it clear how we apply the results of [4].

\subsection{Even characteristic}

If $q$ is even, then the tangent spaces of a pseudo-oval $\mathcal{E}$ all have a $(d / 3-1)$-space in common; the nucleus of $\mathcal{E}$ (see [20, pp. 182]). Since $G$ must fix the nucleus, we have that $G$ acts reducibly in this case. Let $\mathcal{N}$ be the the nucleus of $\mathcal{E}$ and consider the quotient map $\pi$ from $\operatorname{PG}(d-1, q)$ to $\operatorname{PG}(d-1, q) / \mathcal{N}$, and note that the codomain can be identified with $\mathrm{PG}(2 d / 3-1, q)$. The image of $\mathcal{E}$ under $\pi$ is a spread $\mathcal{S}$ of $\operatorname{PG}(2 d / 3-1, q)$ (see [20, pp. 182]). Moreover, we have that $G$ acts transitively on this spread, and by the Andre/Bruck-Bose construction, we obtain a flag-transitive affine plane admitting an insoluble group. By [7], this affine plane is Desarguesian or a Lüneburg plane, so in particular, it follows that $\mathcal{E}$ admits a 2-transitive group. So by [24, §8], we have that $\mathcal{E}$ is an elementary pseudo-oval arising from a conic of $\operatorname{PG}\left(2, q^{d / 3}\right)$.

\subsection{Odd characteristic}

Let $\mathcal{E}$ be a pseudo-oval of $\operatorname{PG}(d-1, q)$, where $q$ is odd. Then each element $E$ of $\mathcal{E}$ is contained in a unique $2 d / 3-1$-subspace $T_{E}$ of $\operatorname{PG}(d-1, q)$ which is called the tangent space at $E$. By [20, pp. 182], each point of $\operatorname{PG}(d-1, q)$ is contained in 0 or 2 tangent spaces of $\mathcal{E}$.

Theorem 3.1. Let $q=p^{f}$ where $p$ is an odd prime, let $d$ be an integer divisible by 3. If an insoluble subgroup $G$ of $\mathrm{PGL}_{d}(q)$ acts transitively on a pseudo-oval $\mathcal{E}$ of $\mathrm{PG}(d-1, q)$, then $\mathcal{E}$ is elementary and is obtained by field reduction of a conic of $\operatorname{PG}\left(2, q^{d / 3}\right)$. 





\section{Alternating group case}

Here $S=A_{r}$ and the vector space $V_{u}(q)$ can be identified with the fully deleted permutation module for $S_{r}$ over $\operatorname{GF}(q)$. We have that $u$ is $r-1$ or $r-2$ (according to whether $p$ does not or does divide $n$ respectively), and $q^{u}=p^{u}=3^{6}, 5^{6}$. Suppose $S=A_{7}, u=6$, and $q=3$. Then $S$ stabilises $\mathcal{M}$ and hence $S$ has a union of orbits on planes of $\mathrm{PG}(5,3)$ of size 14 or 28 . Now $A_{7}$, in its unique irreducible representation in $\mathrm{PG}(5,3)$ has the following orbit lengths on planes (n.b., the exponents denote multiplicities):

$$
\left[35^{2}, 105^{4}, 140^{3}, 210^{4}, 315^{6}, 420^{10}, 630^{6}, 840^{4}, 1260^{15}\right] .
$$

Therefore this case does not arise. Now suppose $q=5$. It can be shown using GAP [11] that the $S$-invariant sets of planes of size 63 or 126 do not cover every point either 0 or 2 times. Therefore this case does not arise.

\section{Cross-characteristic case}

The table below lists the possibilities for this case.

\begin{tabular}{|c|c|c|c|}
\hline$S$ & $d$ & $q$ & $u$ \\
\hline $\mathrm{PSL}_{2}(7)$ & 9 & 3 & 6 \\
$\mathrm{PSL}_{2}(13)$ & 9 & 3 & 6 \\
$\mathrm{PSU}_{3}\left(3^{2}\right)$ & 9 & 5 & 6 \\
\hline
\end{tabular}

Now $\mathrm{PSL}_{2}(13)$ acts transitively on the points of $\mathrm{PG}(5,3)$, and so this case does not arise. Suppose $S=\mathrm{PSL}_{2}(7), u=6$, and $q=3$. Then $S$ stabilises $\mathcal{M}$ and hence $S$ has a union of orbits on planes of $\mathrm{PG}(5,3)$ of size 14 or 28 . Now by using GAP [11] and the unique irreducible representation for $S$ in $\mathrm{PG}(5,3)$, we have that $S$ has the following orbit lengths on planes:

$$
\left[7^{4}, 21^{8}, 28^{12}, 42^{18}, 56^{12}, 84^{100}, 168^{140}\right] .
$$

None of the thirteen $S$-invariant sets of planes of size 28 have each point of $\mathrm{PG}(5,3)$ contained in a constant number $(0$ or 2$)$ of elements of the set. Likewise, of all the six $S$-invariant sets of size 14 , none have each point of $\operatorname{PG}(5,3)$ contained in a constant number of elements of the set. Therefore, this case does not arise.

Now suppose $S=\mathrm{PSU}_{3}\left(3^{2}\right), u=6$, and $q=5$. Then $S$ stabilises $\mathcal{M}$ and hence $S$ stabilises a set of points of size $\left(q^{u}-1\right) /(2(q-1))=1953$. However, by using GAP [11] one can calculate that $S$ has the following orbit lengths on points of $\mathrm{PG}(5,5)$ :

$$
\left[189^{2}, 1008^{2}, 1512\right] .
$$



However in the first case, it is clear that $G$ does not have a subgroup of index 65 . In the second case, we know by [13] that $\mathrm{PSL}_{2}(25)$ has a unique 12dimensional irreducible representation (up to quasi-equivalence) over $\mathrm{GF}(2)$ and it has the following orbit lengths on points:

$$
\left[65,325^{2}, 650,780,1950\right] \text {. }
$$

Let $\mathcal{B}$ be the set of points covered by the pseudo-ovoid $\mathcal{E}$ of $\mathrm{PG}(11,2)$. Then $\mathcal{B}$ has size $\left(q^{d / 4}-1\right)\left(q^{d / 2}+1\right)=\left(2^{3}-1\right)\left(2^{6}+1\right)=455$ and it must be a union of orbits of $S$ as $G$ acts transitively on $\mathcal{E}$. However, 455 cannot be partitioned into the orbit lengths displayed above, and hence this case does not arise.

That leaves us with the Extension field examples. Here we have that $\hat{G} \leqslant$ $\Gamma \mathrm{L}_{d / b}\left(q^{b}\right)$ where $b$ is a divisor of $d$ (where $b \neq 1$ ). If $d / b>2$, We can apply [4, Theorem 3.2] (for $e / b$ even) and [4, Theorem 3.1] (for $e / b$ odd) to $\hat{G} \cap \mathrm{GL}_{d / b}\left(q^{b}\right)$ with parameters $d / b, e / b$, and $q^{b}$ playing the roles of $d, e$, and $q$ respectively. We have the following subcases:

(i) $d / b=4$ and $\Omega_{4}^{-}\left(q^{d / 4}\right) \preccurlyeq \hat{G} \cap \mathrm{GL}_{d / b}\left(q^{b}\right)$;

(ii) $d / b=4, q$ is even, and $\mathrm{Sz}\left(q^{d / 4}\right) \Vdash \hat{G} \cap \mathrm{GL}_{d / b}\left(q^{b}\right)$;

(iii) $d / b=3, q^{d / 3}$ is a square, and $\mathrm{SU}_{3}\left(q^{d / 3}\right) \preccurlyeq \hat{G} \cap \mathrm{GL}_{d / b}\left(q^{b}\right)$.

(i) Let us suppose we have the first case above, where $d / b=4$ and $\mathcal{E}$ admits $\mathrm{P} \Omega_{4}^{-}\left(q^{d / 4}\right)$. Let $J=\mathrm{P} \Omega_{4}^{-}\left(q^{d / 4}\right)$. It is a classical result, but can also be found in [8], that $\mathrm{PSL}_{2}\left(q^{d / 2}\right)$ (where $d>2$ ) has a unique conjugacy class of subgroups of index $q^{d / 2}+1$. Note that $\mathrm{P} \Omega_{4}^{-}\left(q^{d / 4}\right)$ is isomorphic to $\mathrm{PSL}_{2}\left(q^{d / 2}\right)$, and by [14, Proposition 4.3.6], there is a unique conjugacy class of subgroups of $\mathrm{PGL}_{d}(q)$ isomorphic to $\mathrm{PSL}_{2}\left(q^{d / 2}\right)$. Therefore, there is a unique conjugacy class of subgroups of $\mathrm{PGL}_{d}(q)$ isomorphic to $J$.

Let $\varphi: V_{4}\left(q^{d / 4}\right) \rightarrow V_{d}(q)$ denote the natural vector space isomorphism here, and let $\mathcal{Q}$ be an elliptic quadric of $V_{4}\left(q^{d / 4}\right)$ admitting $J$. Let $\alpha$ and $\beta$ be two distinct points of $\mathcal{Q}$. Then $\varphi(\alpha)$ and $\varphi(\beta)$ are $d / 4$-dimensional subspaces of $V_{d}(q)$. Note that $J$ has a unique conjugacy class of subgroups of index $q^{2}+1$ (see [8]), and hence we can assume that the stabiliser of an element $E$ of $\mathcal{E}$ is identical to the stabiliser $J_{\alpha}$. Now suppose we have a third vector $v$ which is neither $\alpha$ nor $\beta$. Then

$$
\left|v^{J_{\alpha}}\right|=\left|J_{\alpha}: J_{\alpha, v}\right|=\left|J_{\alpha}: J_{\alpha, \beta}\right|\left|J_{\alpha, \beta}: J_{\alpha, \beta, v}\right|=q^{d / 2}\left|J_{\alpha, \beta}: J_{\alpha, \beta, v}\right| .
$$

Now $J$ is a Zassenhaus group and so $J_{\alpha, \beta, v}=1$. Therefore

$$
\left|v^{J_{\alpha}}\right|=q^{d / 2} \frac{q^{d / 2}-1}{\operatorname{gcd}\left(2, q^{d / 2}-1\right)}
$$





, Translation planes, Springer-Verlag, Berlin, 1980.

[17] H. H. Mitchell, Determination of the ordinary and modular ternary linear groups, Trans. Amer. Math. Soc. 12 (1911), no. 2, 207-242.

[18] C. M. O'Keefe and T. Penttila, Symmetries of arcs, J. Combin. Theory Ser. A 66 (1994), no. 1, 53-67.

go back

full screen

close

quit

[19] G. Panella, Caratterizzazione delle quadriche di uno spazio (tridimensionale) lineare sopra un corpo finito, Boll. Un. Mat. Ital. (3) 10 (1955), 507-513.

[20] S. E. Payne and J. A. Thas, Finite generalized quadrangles, Research Notes in Mathematics 110, Pitman (Advanced Publishing Program), Boston, MA, 1984.

[21] T. Penttila, Translation generalised quadrangles and elation laguerre planes of order 16, European J. Combin., to appear.

[22] B. Segre, Sulle ovali nei piani lineari finiti, Atti Accad. Naz. Lincei. Rend. Cl. Sci. Fis. Mat. Nat. (8) 17 (1954), 141-142.

[23] M. Suzuki, On a class of doubly transitive groups, Ann. of Math. (2) 75 (1962), 105-145.

[24] J. A. Thas and K. Thas, Translation generalized quadrangles in even characteristic, Combinatorica 26 (2006), 709-732.

[25] K. Zsigmondy, Zur theorie der potenzreste, Monatsh. für Math. u. Phys. 3 (1892), 265-284.

John Bamberg

School of Mathematics and Statistics, The University of Western Australia, 35 Stirling HighWAy, CraWley W.A. 6009, Australia

Department of Pure Mathematics, Ghent University, Galglaan 2, 9000 Gent, Belgium

e-mail: bamberg@cage. ugent. be

Tim Penttila

School of Mathematics and Statistics, The University of Western Australia, 35 Stirling HighWAY, CraWley W.A. 6009, Australia

Department of Mathematics, Colorado State University, Fort Collins, CO 80523, USA

e-mail: penttila@math.colostate.edu 